\title{
APPLICATION OF 3D MEASUREMENT TECHNOLOGY IN URBAN HIGH-RISE BUILDING PLANNING PROJECT
}

\author{
Ang Wang ${ }^{1}$, Wenxiang Han ${ }^{2}$ \\ ${ }^{1}$ School of Civil Engineering, Huanghe Science \& Technology College, Zhengzhou City, Henan, 450000, China \\ 2Zhongyuan University of Technology, Zhengzhou City, Henan, 450000, China \\ Email: wangang1@sina.com
}

\begin{abstract}
Modern high-rise building projects are generally featured by tight schedules, heavy tasks, high technical requirements, small construction sites and other difficulties. As a three-dimensional measurement information management system, BIM (Building Information Modelling) system can realize the integration and establishment of information data in all links of planning and construction management of buildings and generate complete information models so as to improve the efficiency of building planning and project management. Hence, it is widely applied in the field. Based on the practical application of BIM system in a high-rise building project, this paper analyzes the concept of BIM system and puts forward the relative solution of BIM technology for the technical difficulties encountered in planning construction and management, which improves the efficiency of construction management, shortens the duration, saves the cost and produces economic benefits. At the same time, this paper also puts forward the method of measuring data with the 3D scanning technology and improves the efficiency of acceptance.
\end{abstract}

Keywords: BIM; High-Rise Building; Three-Dimensional Model; Three-Dimensional Laser Scanning.

\section{Introduction}

Since the implementation of the reform and opening up policy, the economy in China has developed rapidly, which makes the scale of cities continue to expand and the level of urbanization continue to increase. Meanwhile, industrial development has made more and more people gathered in cities, which makes the urban land resources become increasingly tense, resulting in the sharp increase in high-rise buildings. Economic development, urban expansion and land scarcity are the root causes of the birth of high-rise buildings. Building information modelling (BIM) refers to the establishment of a building model based on the relevant information data of a construction project to realize the simulation of the real information of the building through digital information simulation. It is not just a simple integration of digital information, but also an application of digital information. The emergence of BIM allows the high-rise building projects to update dynamic changes at different stages and provides reliable information basis for the projects, which helps to accelerate project progress, improve project quality and reduce project costs.

Both domestic and foreign scholars have conducted researches on the application of BIM in high-rise building projects. L Lin, J Li et al. (Lin et al., 2016) believed that the application of BIM technology could control the quality of construction more effectively, optimize the allocation of resources and ensure the safety of basic engineering, compared with traditional construction management methods.

Sun Jinlie (Sun, 2015) held that the BIM threedimensional model system could achieve all aspects of planning and visual management of high-rise building construction. Dimensional laser scanning technology is a fully automatic high-precision threedimensional scanning measurement technology that can realize the rapid acquisition of large-scale building 3D coordinate data in a complex environment, which is a fruit of the revolution in the field of mapping (Tang et al., 2013) in addition to the GPS technology (Zhang, 2016). F Wang, H Lin (Wang et al., 2012) proposed a large-scale street building facade mapping technology program based on the ground three - dimensional laser scanning technology and verified the advantages of speediness and accuracy of the technology in detail measurement through the practical application of the production project. However, since the automation degree of the technology in post-data processing was low and the human input was large, there was still a long way to go for the research and development of the post-data processing software.

This paper takes a high-rise building project in Henan Province as an example to explore and analyze the application of BIM three-dimensional building model in high-rise building planning 
projects and discusses the possibility of the application of the three-dimensional laser scanning technology at the completion and acceptance stage of projects.

\section{Project Overview}

A high-rise building project in a city in Henan province covers an area of $24874.2 \mathrm{~m} 2$, with a total construction area of $145917.2 \mathrm{~m} 2$ (aboveground area of about $114179.2 \mathrm{~m} 2$, underground area of about $31738 \mathrm{~m} 2$ ). It all has three buildings, including two 26-floor office buildings and one 29-floor office building. The height of the main body is $99.8 \mathrm{~m}$, equipped with a 2-storey basement, as shown in figure 1 .

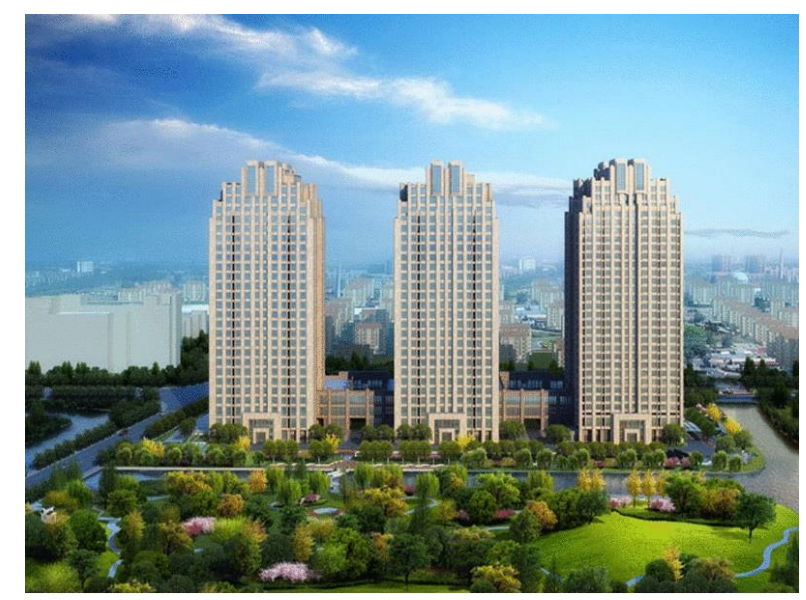

Figure 1: Project effect design preview

\section{Project Technical Difficulties and the Application of BIM in The Project}

\section{Project difficulty overview}

Due to the large volume of the project and the complexity of the design, much manpower, material resources and financial resources are input. Besides, some new technologies and materials are involved in the project, leading to many problems in the design and construction of the project. In the early stage of the project, due to the frequent changes in project design and large amount of drawings, the use of traditional two-dimensional drawings will lead to slow progress and duration delays. Moreover, because of the complexity of the process and the high cost of new materials, failure in a complete design of the building structural scheme can cause a waste of structural design. With the accuracy of the two-dimensional drawings yet to be verified, the design rationality check requirements are getting higher and higher. As a result, the design must be deepen in the early stages. Attention should be paid to the cooperation between different types of work in high-rise building construction because crossprocess contradictions will not only cause delays in the construction, but also affect the quality of the completed construction project. In a short duration, the BIM technology can be applied to build a threedimensional model diagram so as to reduce the economic losses and delays caused by design changes, cross processes and repeated purchase of materials, implement monitoring progress and ensure the quality of the project.

\section{The application of BIM}

Building information model BIM technology (Zhu et al., 2010) is a data model established based on the three-dimensional digital information technology that integrates various relevant information in engineering projects. The main role of BIM (Zhang et al., 2013) is to establish a three-dimensional model to minimize as far as possible or even completely eliminate the foreseeable or unpredictable problems in the project, so as to fundamentally solve the information management problems of the project from the early development and design stage to the late construction and operation phase to achieve a thorough and comprehensive information management of the entire project process.

\section{On material calculation}

As the main structure of the building is complex and irregular, it is difficult to calculate the project amount. Relying on manual calculation will not only slow down the progress, cost a lot of manpower, but is also likely to cause errors, resulting in unnecessary economic losses. On the contrary, the data of the BIM database can timely and accurately provide the data information required to complete all levels of management of the project as well as rapidly and precisely calculate the project amount.

Through the establishment of the BIM information model, all the material lists can be directly obtained, which not only reduces the possibility of material calculation errors, but also helps to effectively draw up related material procurement plans, so as to enhance the construction budget accuracy and efficiency, avoid unnecessary waste and improve profitability. Since the traditional two-dimensional drawings cannot quickly and accurately obtain massive engineering data, which leads to the failure of construction enterprises to achieve fine management, the method of using empiricism to manage comes out. However, this method will also cause great errors, resulting in a lot of waste and losses. Hence, BIM technology, which can accurately and rapidly obtain basic project data, comes up to solve this problem.

By applying this technology, construction units can develop reasonable plans to reduce unnecessary waste of resources and logistics.

\section{On construction image progress and large mechanical layout}

The construction progress of the construction site and the overall situation of a variety of 
mechanical configuration (Kim et al., 2013) can be intuitively expressed through establishing a three dimensional information model applying Revit (An et al., 2015) and exporting the relevant renderings with the 3dMax software.
Meanwhile, the building's roaming ( $\mathrm{Xu}$ et al., 2013) video is made, through which the inside structure of the building can be viewed, to fully grasp the internal situation of the building, as shown in Figure 2.

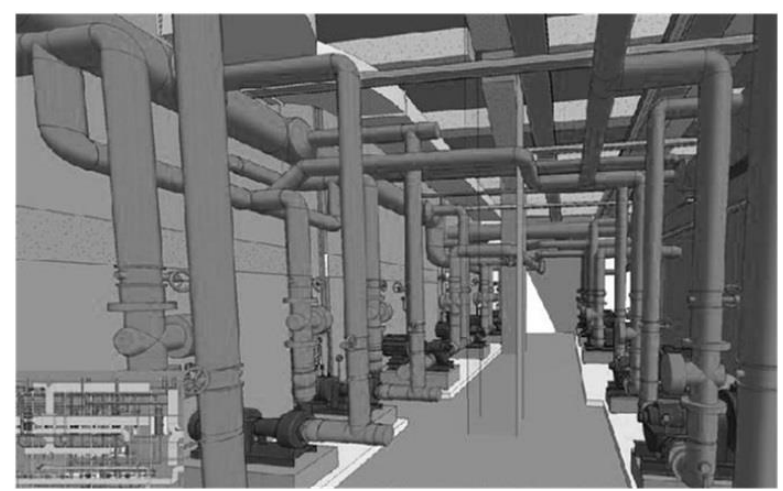

Figure 2: The internal roaming map of the three-dimensional model building

By making use of the system parameters within the database, decision-making at the construction stage can be carried out quickly. The core of BIM technology is that data will go through real-time dynamic changes when the construction state of the building is changed to achieve high quality management of the project and realize data sharing through the information network and the maximum utilization of resources.
Figure 3 shows the virtual construction functions of the project.

Through the combination of BIM technology and construction solutions, construction simulation and live video detection, owners and project leaders can intuitively compare the construction plan and the actual progress anytime and anywhere, which reduces the frequency of the occurrence of the building's quality problems.

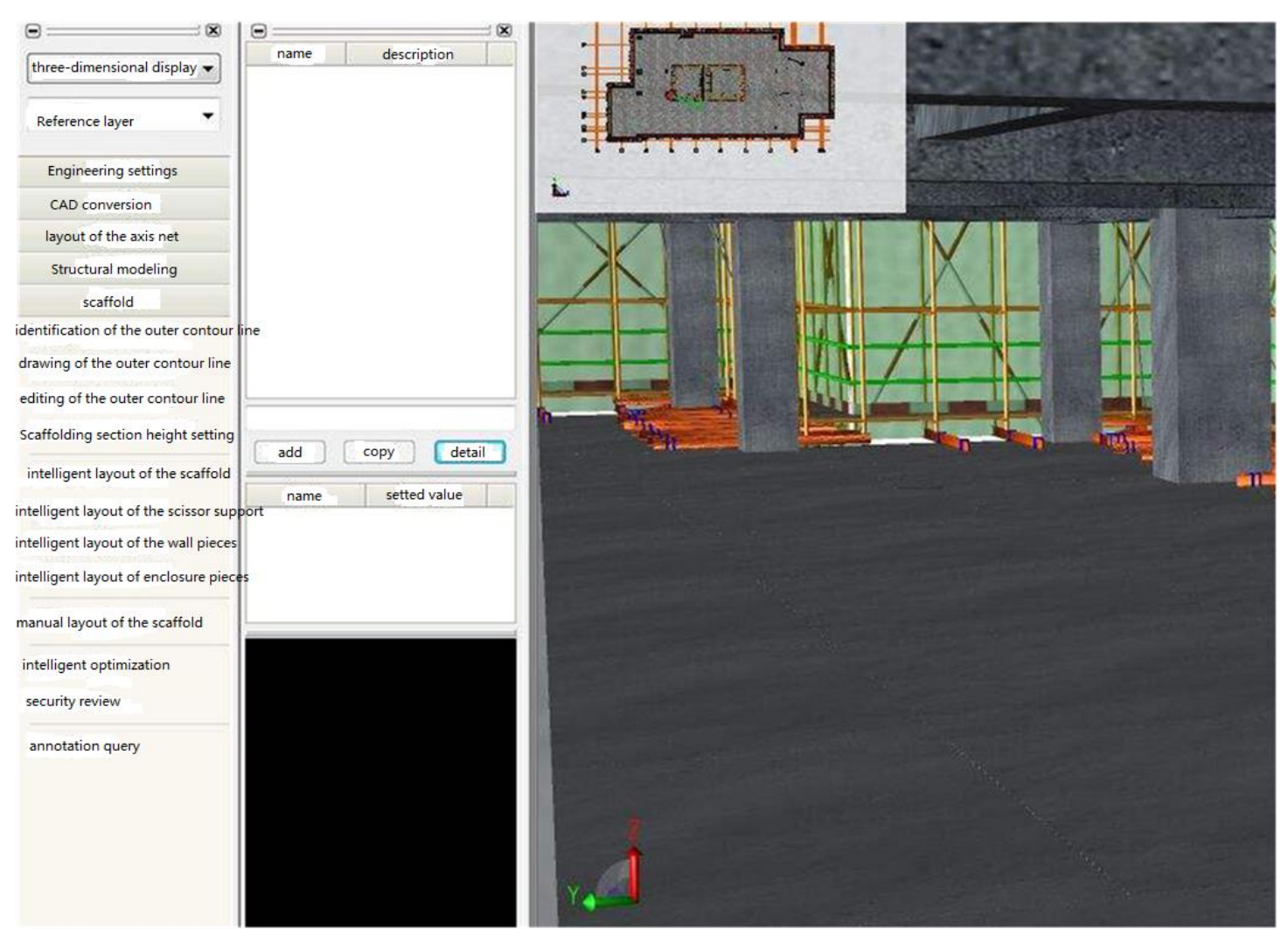

Figure 3: Virtual construction coordination diagram 
Since the 3D model can give people the most intuitive feeling, anyone can have the access to the construction schedule and equipment configuration problems and modify the model (Gao et al., 2016) after finding out problems to reduce unnecessary rework.

\section{On the layout of the construction site}

In the actual construction, due to the material stacking, the parking of vehicles and the piling up of temporary facilities, it is likely to cause layout chaos in the construction site, resulting in the problems of road congestion, material loss and inadequate processing site, etc. Moreover, temporary changes will not only waste a lot of manpower and time, but also cause economic losses. BIM system can establish building models as well as models for the layout of the materials, vehicles and temporary facilities at each stage of the project to simulate the real situation before construction to visually show the various problems that might be encountered so as to improve the layout and avoid these problems in advance.

\section{On Collision detection}

Pipeline collision is inevitable in the construction process. However, it not only delays the time, but also affects the original overall design to change the pipeline circuit by design changes after the occurrence of the problem. The underground part of the project in this study focuses on a large number of pipelines, which are intricate and require high construction accuracy, with frequent changes, lots of mechanical connections, embedded parts and reserved holes, bringing great difficulties to the integrated pipeline layout. Through the application of the BIM software, the water, electricity and heating and ventilation models can be established.

Besides, the possible location of collision can be found through the collision detection. Hence, the design can be improved in advance, the reasons for the collision of the project can be checked and different work can be coordinated and organized to reduce construction delays and rework.

The specific operational processes are: professional modelling, collision detection, collision reporting, collision reporting, high-speed principle development, model validation and two-dimensional mapping.

\section{On tendering}

The BIM three-dimensional model after 3DMAX rendering can give people an intuitive visual impact, leaving a more realistic sense of publicity to the owners.

Hearing the project introduction, the owners can find the corresponding embodiment from the threedimensional model. Meanwhile, the three dimensional model map can show the high-end technologies of the project to the owners, thereby enhancing the probability of winning the bidding.

\section{Application of 3D Laser Scanning Technology in Project Completion Acceptance}

As the three-four layers of the construction are hollow, with irregular structure arrangement, it is difficult to use conventional measurement methods for field data collection. Therefore, the threedimensional laser scanning technology is applied to complete the completion acceptance.

\section{Overview of 3D Laser Scanning Technology}

Three-dimensional laser scanning (Li et al., 2012), also called "live-action copy technology", is a technology which can go deep into a variety of complex environments for high-precision, largescale scanning operation to quickly get the threedimensional coordinate data on the surface of the measured object. It can be applied to the point cloud data collection on the surface of a variety of objects and is rapid and accurate. Hence, it is widely applied in building surveying and maintenance, appearance structure 3D modelling, pavement topography measurement, underground structure measurement, etc. Its advantage is that it can carry out rapid and accurate measurement without touching the measured object and can obtain data with high accuracy of the objects with complex internal structure which are difficult to measure. Besides, the cloud data obtained by scanning can be directly transformed into a BIM model, which can not be realized by traditional measurement methods.

\section{Three - dimensional laser scanning measurement data acquisition}

The project uses RIEGL VZ-1000 threedimensional laser scanner, with a nominal operation distance of 1400 meter and a reflectivity of $90 \%$.

The following two scanning methods are used to carry out fine scanning on the three buildings: First, single station absolute orientation mode.

This mode needs to set the scanning station and target at the mapping base point, and also needs to complete the scanning of the corresponding parameters and carry out the scanning work; second, no target relative orientation pattern. This mode can directly complete the scanning of the corresponding buildings and the operation is simple.

When scanning the site coordinates, the internal set GPS functions can be developed, which is easy to improve the work efficiency.

Twenty six stations were scanned in each of the three buildings and 10 targets were affixed to the building curtain walls or to high objects with obvious features such as poles. 
Meanwhile, the total station is used to measure the coordinates of the target and the station. Figure 4 shows collection sites of building 1 .

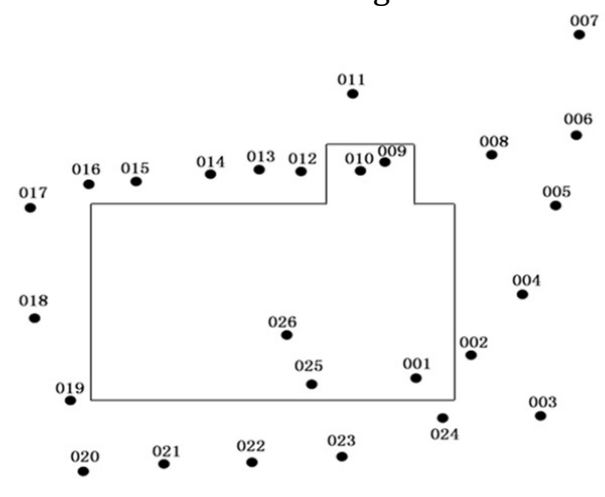

Figure 4: Field data collection site diagram of building 1

\section{Calculation of building area}

After scanning the engineering buildings, the three-dimensional point cloud data are denoised, repaired and registered, appropriate slice thickness is designed and slicing processing are performed on each layer. With the horizontal plane $X-Y$, the characteristic lines for each layer are drawn after data extraction of the obtained feature points to get the building area of each layer. Through outputting the characteristic lines of each layer from the drawing exchanging software to the CAD software, the line map of the building can be drawn, so as to get the building area.

\section{D completed measurement data analysis}

The ground three-dimensional laser scanner is used for observation and all point cloud data are connected after the second filtering and denoising processing. Besides, a comparison between the calculated floor area and the actual area suggests that the error is small, which is only $4.3 \mathrm{~m} 2$.

The three-dimensional laser scanner can input each floor map into CAD software to obtain threedimensional images (Lu et al., 2012) and draw the most intuitive conclusion through the image comparison with the BIM three-dimensional building map, with high applicable values.

\section{The Influence of 3D Measurement Technology on Construction Cost}

Influence of BIM Building Information Model on Construction Cost

BIM three-dimensional information construction model has good technology data compatibility, and can quickly and accurately integrate information.

Therefore, its degree of three-dimensional visualization is higher than other technologies, which promotes the leapfrog development of the integration of building information.
(1) Visualization analysis of the threedimensional model can help to find structural problems in the pre-design stage to modify the model so as to reduce the rework problem.

(2) The modified model can be combined with other professional models for collision analysis and the analysis results can be used to solve the professional problems, to avoid the impact of different construction work.

(3) By the construction simulation at the critical stage of the project, a planned period through the critical stage can be ensured to achieve the desired results to ensure the smooth progress of the project.

(4) BIM model records all the building information, which can be quickly taken at the completion and acceptance stage. Besides, it can also be used for post-construction maintenance and property information maintenance, which brings continuity to the owners to maximize their rate of return on investment costs.

In summary, BIM technology can reduce the economic losses and delays caused by design changes, improve the production efficiency of the site, shorten the construction period and ensure the quality of the project, thus saving the construction cost.

\section{The influence of 3D laser scanning technology on construction cost \\ Compared with the traditional mapping method,} three-dimensional laser scanning technology is efficient and safe and can quickly, continuously and automatically obtain high-precision, high-density three-dimensional data. The obtained building space information can be directly converted into point cloud data which can be processed, and can be imported into BIM and other engineering design software for more in-depth management and use.

Moreover, it overcomes the limitation of measurement and can scan at any time to obtain high-precision building three-dimensional coordinate data and capture the details of the building information. Therefore, three-dimensional laser scanning technology greatly shortens the time of completion and acceptance, reduces the lab or intensity, improves the efficiency of the field work, and reduces the cost of building completion acceptance.

\section{Conclusion}

To sum up, we can see that BIM threedimensional building information model technology has brought benefits for the research, design, construction and operation and maintenance stage, such as improving the design quality, the level of engineering management information, the workability, project management efficiency and 
reducing human, material, material consumption, duration delay, etc.

It reduces the cost of materials during construction and the unnecessary waste of resources due to changes in drawings and so on, saving construction costs.

Three-dimensional laser scanning measurement technology saves time and effort, speeds up the completion of the acceptance and reduces costs, comparing with traditional two-dimensional completion detection methods when there is an increase in the shape of the building and planning management is complicated. The combination of these two technologies can speed up the city construction and optimize the layout of the city, driving the city's economic efficiency. The changes brought by the three-dimensional building information model technology and threedimensional laser scanning technology to the highrise building engineering projects are revolutionary.

The application and development of any new technology requires a lot of theoretical and practical support and we believe that these two technologies will play a greater role in the future urban high-rise building planning projects.

\section{References:}

[1] An Pei, Yu Dihua, Ai Xinying, et al., 2015. Research and Application of 3D Quantity Calculation Based on BIM in Super Tall Building Engineering. Construction Technology, 44(23):15-18.

[2] Gao Cheng, Qu Haiyan, Zhao Xuexin, et al., 2016. Study and Application of BIM in Construction of Some Super High-rise Project. Steel Construction, 31(6):88-91.

[3] Kerosuo H, Miettinen R, Paavola S, et al., 2015. Challenges of the expansive use of Building Information Modeling (BIM) in construction projects. Prod, 25(2):289-297.

[4] Kim H, Anderson K, Lee S H, et al., 2013. Generating construction schedules through automatic data extraction using open BIM (building information modeling) technology. Automation in Construction, 35(2):285-295.

[5] Lin L, Li J, Huang M, et al., 2016. The Application and Exploration of the Foundation Construction Management in High-Rise Building Based on BIM Technology. International Conference on Smart City and Systems Engineering. IEEE Computer Society, 304-307.

[6] Li Yadong, Lang Haochuan, Wu Tianhua, 2012. Appication of Field Scanning Combined with BIM Technology in Engineering Impementation. Construction Technology, 41(18):19-22.

[7] LU Cui-hua, CHEN Xiu-ping, ZHANG Dong-ming, 2012. The Building 3D modeling method Based on 3D Laser Scanning Technology. Science Technology and Engineering, 12(10):2410-2414.

[8] Sun Jinlie, 2015. Application of BIM Visualization Technology in Super-high-rise Building Structure Design. Architecture Engineering Technology \& Design, (32).

[9] Tang Kun, Hua Xianghong, Wei Cheng , et al., 2013. The Experimental Study of Buildings Deformation Monitoring Method Based on 3D Laser Scanning. Journal of Geomatics, 38(2):5455.

[10] Wang F, Lin H, Changhui L I, 2012. Application of Terrestrial 3D Laser Scanning Technology in Urban Surveying. Bulletin of Surveying \& Mapping, 9(5):47-49.

[11] Xu N, Wang L, 2013. Study on Technical Disclosure in Construction Based on BIM. Applied Mechanics \& Materials, 438-439:1698-1701.

[12] Zhang Guanghui, 2016. Application of GPS Surveying Technology in High-rise Building Construction. Engineering Technology: Full text, (7): 00242-00242.

[13] Zhang L, Zhang X, Ma T, 2013. Management of Construction Schedules Based on Building Information Modeling Technology. 236:81-88.

[14] Zhu L, Lian W S, Wang F, 2010. BIM Digital Technology in the Construction Industry in the Field of Application. Advanced Materials Research, 143-144:1375-1379. 